\title{
A Novel Active Bouncer System for Klystron Modulators with Constant AC Power Consumption
}

\author{
F. Cabaleiro Magallanes, D. Aguglia \\ CERN, Geneva, Switzerland \\ P. Viarouge, J. Cros \\ LEEPCI Lab., Quebec, Canada \\ C. de Almeida Martins \\ ESS, Sweden
}

Keywords: Bouncer System, Klystron

\begin{abstract}
This paper presents the principles and design methodologies of a novel active bouncer system, to be implemented in a transformer-based klystron modulator, which is able to meet two different objectives: 1. Regulate the output pulse voltage flattop, and 2. Attenuate the power fluctuation withdrawn from the AC network. This solution allows the utilization of a standard constant voltage / constant current power supply as a capacitor charger. The solution consists of a 4-quadrant switching converter placed in series with the main capacitor bank (forming a unique element in parallel with the capacitor charger), controlled with specific feed-back loops to achieve the two objectives. The complete design method, including a numerical optimization, of the whole system, is presented in the paper. Analyses of the compromises between the active bouncer specifications and the other modulator sub-components design is presented as well.
\end{abstract}

Presented at: IEEE pulsed power and plasma science conference (PPPS),

San Francisco, USA, June 2013

Geneva, Switzerland

November, 2014 


\title{
A Novel Active Bouncer System for Klystron Modulators with Constant AC Power Consumption
}

\author{
Francisco Cabaleiro Magallanes ${ }^{1 \& 2}$, Member, IEEE, Davide Aguglia ${ }^{1}$, Member, IEEE, \\ Philippe Viarouge ${ }^{2}$, Member, IEEE, Carlos de Almeida Martins ${ }^{3}$, Member, IEEE, Jérôme Cros $^{2}$, Member, IEEE \\ ${ }^{1}$ CERN - European Organization for Nuclear Research, Technology Dept., Electric Power Converter Group \\ CH-1211 Geneva 23, Switzerland Emails : fcabalei@cern.ch, davide.aguglia@cern.ch \\ ${ }^{2}$ LEEPCI Lab., Electrical and Computer Eng. Dept., Laval University, GIK 7P4 Quebec (QC), Canada \\ Emails : philippe.viarouge@gel.ulaval.ca, jerome.cros@gel.ulaval.ca \\ ${ }^{3}$ European Spallation Source (ESS), Sweden Email : Carlos.Martins@esss.se
}

\begin{abstract}
This paper presents the principles and design methodologies of a novel active bouncer system, to be implemented in a transformer-based klystron modulator, which is able to meet two different objectives : 1 . Regulate the output pulse voltage flattop, and 2. Attenuate the power fluctuation withdrawn from the AC network. This solution allows the utilization of a standard constant voltage / constant current power supply as a capacitor charger. The solution consists of a 4-quadrant switching converter placed in series with the main capacitor bank (forming a unique element in parallel with the capacitor charger), controlled with specific feed-back loops to achieve the two objectives. The complete design method, including a numerical optimization, of the whole system, is presented in the paper. Analyses of the compromises between the active bouncer specifications and the other modulator sub-components design is presented as well.
\end{abstract}

\section{Nomenclature}

$D P A B \quad$ Dual Purpose Active Bouncer

PT Pulse Transformer

FTS Flat-Top Stability

$C_{\text {main }} \quad$ Modulator main capacitor bank

$I_{c h} \quad$ Capacitor charger output current

$V_{c h} \quad$ Capacitor charger output voltage

$V_{p} \quad$ Primary voltage

$V_{b_{\max }} \quad$ Active bouncer maximum output voltage

$R_{k l y} \quad$ Equivalent klystron resistance

$R_{t} \quad$ Transformer ratio

\section{INTRODUCTION}

A new linear accelerator under study at CERN, the Compact Linear Collider (CLIC) [1] [2], requires the design of a new klystron modulator capable of producing $150 \mathrm{KV}$ and $160 \mathrm{~A}$ square shaped pulses with a repetition rate of $50 \mathrm{~Hz}$ and a flat-top length of $140 \mu \mathrm{s}$ [3]. The whole complex will include 1638 modulators of 24 MW peak power each, synchronously operated. With this operating mode, a total pulsed peak power of $39.312 \mathrm{GW}$ must be produced, equivalent to $294.84 \mathrm{MW}$ of average power consumption. The utility grid can tolerate only a few percent of power fluctuation and, thus, the klystron modulators system must be designed such that only a constant power (294.84 MW) is withdrawn from the AC network. This power fluctuation can be reduced either by passive or active solutions. Typical capacitor discharge based klystron modulators are already attenuating this effect by a considerable amount (typically $90 \%$ of power fluctuation attenuation). However, because of design constraints, the capacitor bank cannot ensure $100 \%$ attenuation of the power consumption fluctuation from the AC network. Therefore, additional active or passive solutions must be foreseen in order to resolve this issue. In addition to this, the pulse requirements also present a technical challenge (see Table I).

At CERN, one of the modulator topologies under study, and subject of this paper, is based on a monolithic pulse transformer, and integrates an active compensation for the power fluctuation issue.

\section{NOVEL DUAL PURPoSE ACTIVE BOUNCER (DPAB)}

\section{A. Topology overview}

A classical monolithic modulator topology is shown in Fig. 1a). It is composed of a medium voltage AC/DC converter which slowly charges a capacitor bank, and a main MV switch which discharges the capacitor bank through a step-up pulse transformer. This system allows a certain degree of decoupling of the pulsed output power from the utility grid. A bouncer circuit is used to control the secondary pulsed voltage (mainly to compensate the voltage droop on $C_{\text {main }}$ during the pulse). Due to the charge and discharge of the main capacitor bank during normal operation, a DC voltage $V_{c h}$ fluctuation at the output of the charger can be observed. Ideally, the capacitor

TABLE I

CLIC Modulator's OUTPUT PULSE SPECIFICATION

\begin{tabular}{cccc}
\hline \hline Nominal pulse voltage & $V_{s n}$ & 150 & $k V$ \\
Nominal pulse current & $I_{s n}$ & 160 & $A$ \\
Pulse peak power & $P_{\text {mod_out }}$ & 24 & $M W$ \\
Rise \& fall times & $t_{\text {rise }}, t_{\text {fall }}$ & 3 & $\mu s$ \\
Settling time & $t_{\text {set }}$ & 5 & $\mu s$ \\
Flat-top length & $t_{\text {flat }}$ & 140 & $\mu s$ \\
Repetition rate & $R E P R$ & 50 & $H z$ \\
Voltage overshoot & $V_{\text {ovs }}$ & 1 & $\%$ \\
Flat-Top Stability & $F T S$ & 0.85 & $\%$ \\
\hline \hline
\end{tabular}




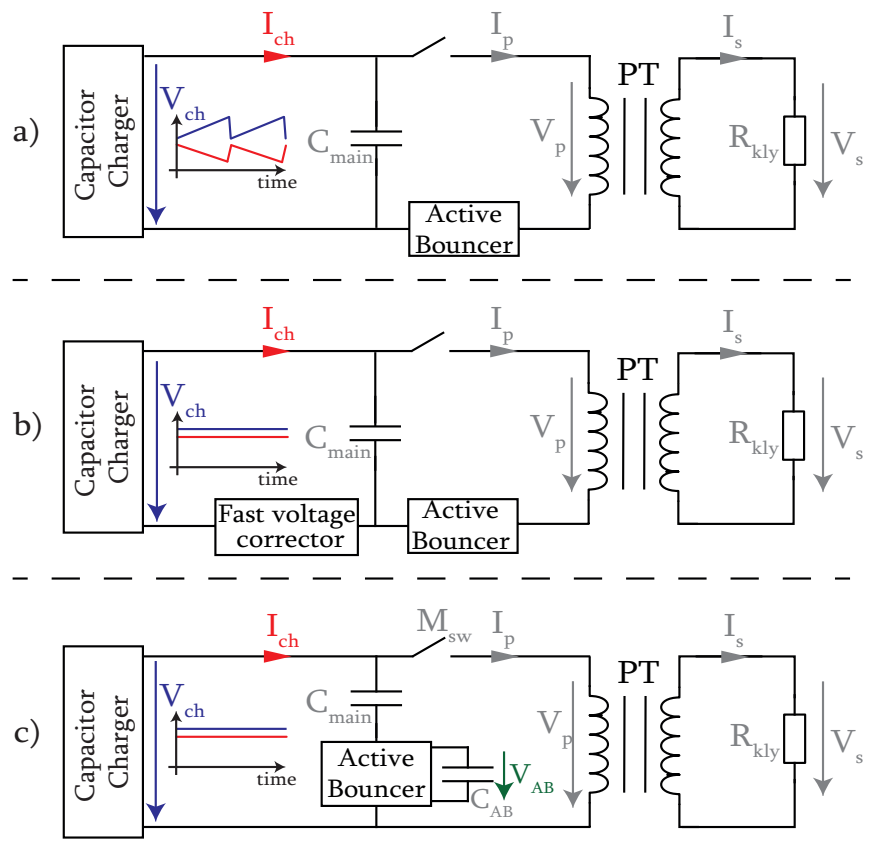

Fig. 1. a) - Classical monolithic modulator topology, b) - with additional fast voltage corrector for AC power fluctuation attenuation, and c) - Dual purpose active bouncer.

charger can be operated such that it follows a current reference computed as $I_{c h_{r e f}}=\frac{P_{a v}}{V_{c h}}$, shown on left of Fig. 1a). However, medium voltage capacitor chargers present a relatively low current bandwidth and, as a consequence, this strategy does not allow an operation at constant DC power consumption at the chargers output (AC and DC power fluctuations at the charger's terminals are directly linked in direct converters, which have no intermediate storage).

A solution consists in trying to drastically increase the current bandwidth of the capacitor charger. However, due to the medium voltage output ratings, this is not an economically interesting solution, which places all the design complexity on the charger system.

Two alternative solutions are currently under study and are proposed in Fig. 1b) \& c). In Figure 1b) a fast voltage corrector is inserted in series to the capacitor charger with the objective of keeping constant the voltage $V_{c h}$. In this way the charger operates at constant DC voltage, current and power consumption, whereas the fast voltage corrector is rated at a fraction of the nominal charging voltage only. This topology is under evaluation and competes with the topology presented in Fig. 1c), equipped with a dual purpose active bouncer (DPAB). The latter topology is the subject of this paper.

Topology of Fig. 1c) utilizes an active bouncer connected in series with the main capacitor bank, forming a parallel branch to the medium voltage capacitor charger. This topological modification makes possible using the DPAB to compensate for the capacitor and transformer voltage droop during the pulse as in a classical topology, as well as the regulation to a fixed value of the charging voltage $V_{c h}$ in order to achieve a constant AC power consumption. As for topology of Fig. 1b), this solution simplifies the requirements of the medium voltage capacitor charger, making possible the use of a standard and commercially available constant DC power supply.

\section{B. DPAB ideal operation}

Depending on the selection of the primary, or charging, voltages $\left(V_{p}\right.$ or $\left.V_{c h}\right)$ and the main capacitor bank value $C_{\text {main }}$, the active bouncer must be able to compensate a given minimum voltage droop to guarantee a correct operation of the modulator. Conversely, given a switches technology (e.g. $600 \mathrm{~V}, 1.2 \mathrm{kV}$ or $1.7 \mathrm{kV}$ IGBTs) or active bouncer topology (e.g. multilevel), the amount of voltage compensation is fixed and primary voltage and capacitor $C_{\text {main }}$ have to be selected, or optimized, accordingly. An idealized operation of the DPAB (Fig. 1c)) during one whole operating cycle (20ms here) is depicted in Fig. 2.

One can identify two operating modes depending on the status of the main switch $M_{s w}$ :

1) Discharging / pulsing operation $\left(M_{s w}\right.$ closed) : the current positively flows into the DPAB (following positive definition in Fig. 1c)). As $C_{\text {main }}$ discharges the active bouncer voltage increases to compensate the droop and regulate the output voltage $V_{s}$. To exploit a maximal voltage excursion the topology can be 2-Quadrants, offering twice the DC-bus voltage of the active bouncer $2 V_{A B}$.

2) Charging operation $\left(M_{s w}\right.$ open) : in this mode the current flows through the active bouncer in the opposite direction (charging current), and the voltage decreases in order to keep a constant charging voltage $V_{c h}$ while $C_{\text {main }}$ is charging.

From this analysis one concludes that the active bouncer should be based on 4-Quadrant topologies. Furthermore notice the zero average power flow through the active bouncer during one cycle (Fig. 2). This demonstrates that, in principle, no additional power converter is (energetically speaking) necessary to supply $C_{A B}$.
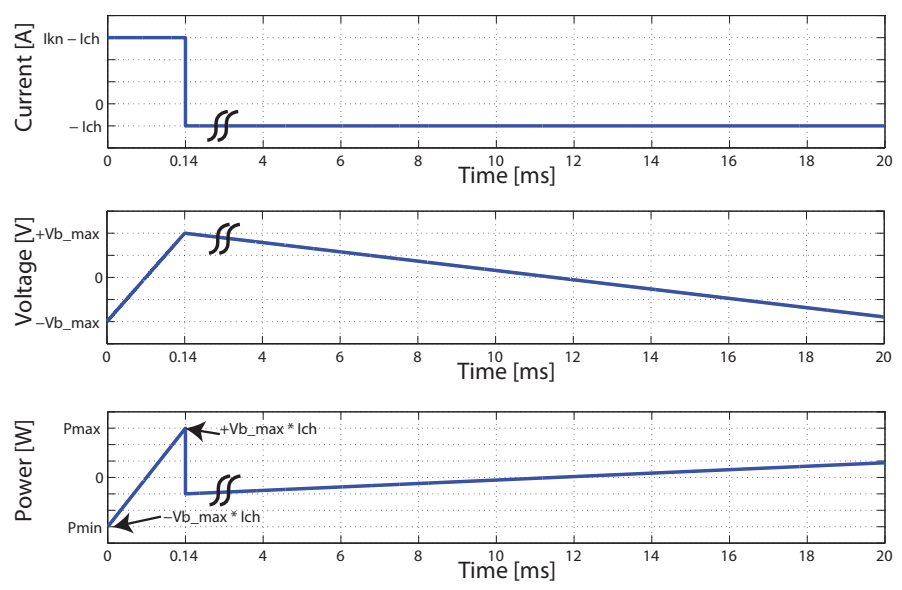

Fig. 2. Ideal current, voltage, and power flow of the DPAB. 


\section{DESIGN COMPROMISES \& NEED FOR AN INTEGRATED SYSTEM APPROACH}

For a given specification in terms of output voltage and $\mathrm{AC}$ power quality, the required specifications of the active bouncer depend on the design of all the other modulator sub-components (mainly the charger, $C_{\text {main }}$, and the pulse transformer). For instance, if the main capacitor bank value $C_{\text {main }}$ is increased, the requirements in voltage excursion from the DPAB is decreased in order to obtain the same performances. On the other hand, if the voltage excursion $V_{A B \max }$ is kept unchanged and $C_{\text {main }}$ increased, higher large signal bandwidth from the active bouncer can be achieved, increasing the global modulator performances. However, an increase of $C_{\text {main }}$ modifies the physical size of the capacitor bank and its stored energy (with implications in overall volume, cost, and safety issues).

Similarly, depending on the PT characteristics (i.e. transformer droop during the pulse), the active bouncer requirements are varying as well. If the voltage droop in the transformer during the pulse increases, it would be necessary to increase the active bouncer output voltage ratings and/or the size of the main capacitor bank. Notice that the DPAB has to regulate the output voltage during the pulse and stabilize the charging voltage in order to reduce the AC power fluctuation. During the pulse operation these two objectives are in contrast, as the $V_{c h} \neq V_{s} R_{t}$ due to the PT droop. As a consequence, this droop influences the power fluctuation during the pulse.

Either the capacitor charger or the DPAB can contribute to the AC power fluctuation active attenuation. Therefore, there is a compromise to be found between the bandwidth specifications of this two elements [4].

With the purpose of studying the influence of each modulator sub-component, in view of deriving the optimal specifications for the whole system, several simulations with sweeps on different parameters values have been carried out. The results from these simulations considering CLIC specifications are presented in the following subsections.

\section{A. Influence of capacitor charger bandwidth}

To study the influence of the DPAB and the charger voltage bandwidths specifications, a simulation model composed of an ideal transformer model with no droop (implying a single control objective for the active bouncer, constantly regulating $V_{p}=V_{c h}=V_{s} R_{t}$ during the whole cycle) with a primary voltage of $10 \mathrm{kV}$ and a DPAB DC-Bus voltage of $1 \mathrm{kV}$ was imposed. The main capacitor bank size is dimensioned considering a total droop of $1600 \mathrm{~V}$ across the main capacitor bank during one pulse. Several simulations where performed by sweeping the active bouncer bandwidth from $10 \mathrm{kHz}$ to $500 \mathrm{kHz}$, whereas the charger bandwidth was made vary from $500 \mathrm{~Hz}$ to $10 \mathrm{kHz}$. Figures 3 and 4 show the Flat-Top Stability (FTS) of the output pulse and the power fluctuation, respectively.

One observes that the active bouncer bandwidth presents the most important influence in the system performances (FTS and AC power fluctuation). The capacitor charger bandwidth

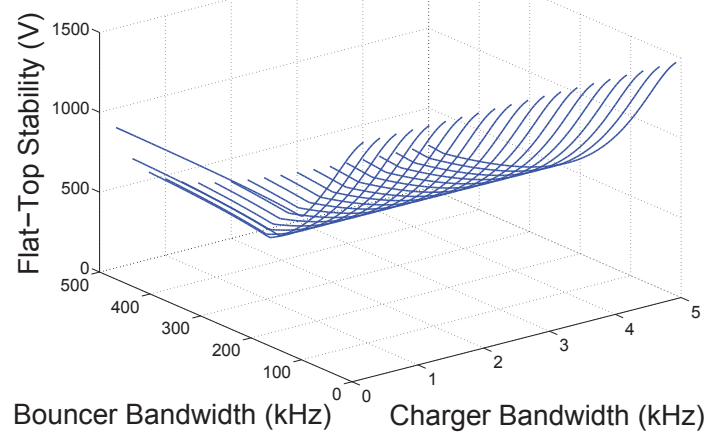

Fig. 3. Voltage fluctuation versus system bandwidths.

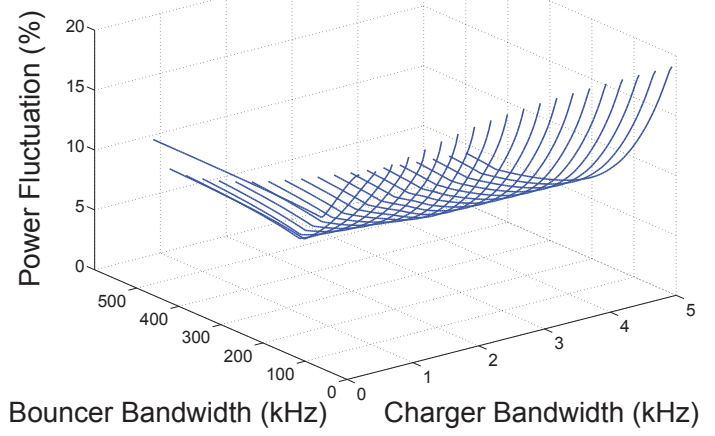

Fig. 4. Power fluctuation versus system bandwidths.

seems also to have an important effect up to a frequency of $1.5 \mathrm{kHz}$ to $2 \mathrm{kHz}$. From this frequency on, a further increase of its bandwidth presents very small increase in global performances.

\section{B. Influence of main capacitor bank size}

As in the last sub-section, several simulations have been carried-out by sweeping the active bouncer voltage bandwidth for several main capacitor bank values. A fixed bandwidth of $800 \mathrm{~Hz}$ has been imposed for the capacitor charger. Figures 5 and 6 show the results in terms of FTS and AC power fluctuation. One can notice that an increase of $22 \%$ in the size of the main capacitor bank (from $225 \mu \mathrm{F}$ to $275 \mu F$ ), for achieving an absolute FTS of $1 \mathrm{kV}$ and a $10 \%$ of power fluctuation, the active bouncer bandwidth requirement is reduced from $100 \mathrm{kHz}$ to $60 \mathrm{kHz}$.

\section{Influence of the pulse transformer droop}

During the pulse some voltage droop appears across the winding resistances and leakage inductance of the pulse transformer [5]. To illustrate its influence on the global modulator performances, the droop phenomena has been emulated in simulations by considering the simplified circuit of Fig. 9, where the voltage droop across $R_{w}$ can be tuned by modifying 


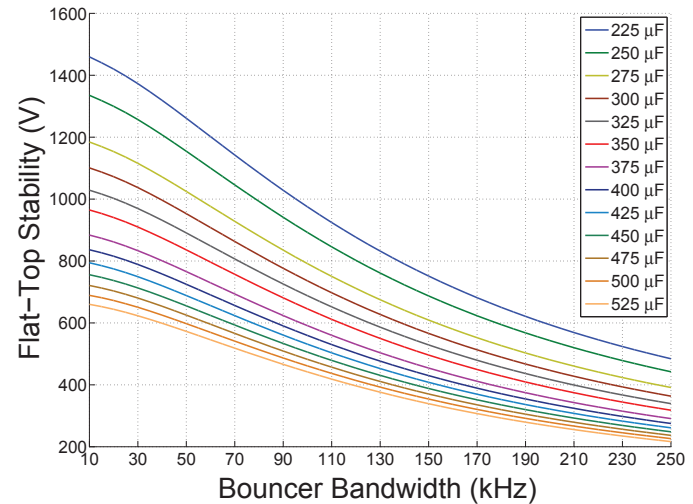

Fig. 5. Influence of capacitor bank size and active bouncer bandwidth on the FTS.

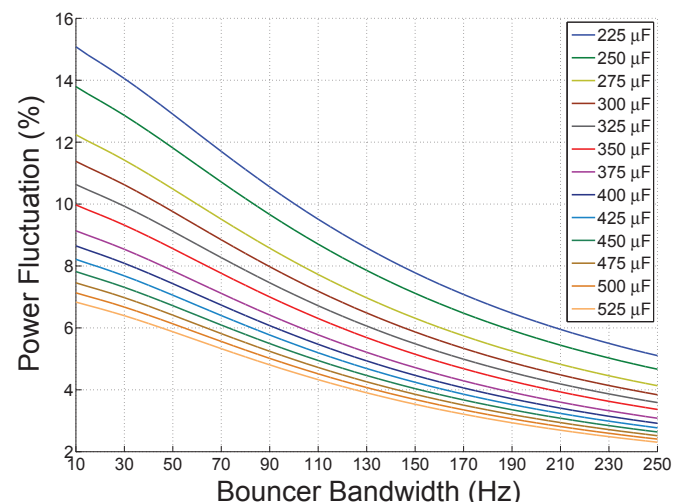

Fig. 6. Influence of capacitor bank size and active bouncer bandwidth on the $\mathrm{AC}$ power fluctuation.

the magnetizing inductance value $L_{m}$ and/or $R_{w}$. Figures 7 and 8 show the influence of the transformer voltage droop during the pulse and the active bouncer bandwidth in terms of FTS and power fluctuation. One can notice a very important impact of the transformer voltage droop on both performances. Notice how the pulse transformer droop greatly affects the FTS and $\mathrm{AC}$ power fluctuation. By designing a low droop pulse transformer the required voltage bandwidth of the DPAB is decreased.

\section{Integrated design approach illustration}

The reciprocal influence of modulator sub-components performances demonstrates the need for an integrated design approach in order to find the optimal design of the system. Due to the complexity of this task, a non-linear constrained numerical optimization-based design procedure can be adopted [6] [7] [8]. This has been carried out on a simplified system depicted in Fig. 9 for illustrative purposes. The active bouncer has been modelled as a single closed-loop transfer function with a tunable second-order dynamics (bandwidth and damping factor). For this example the DPAB switching harmonic attenuation (output filter design) is neglected. During the pulse $V_{s}$ is corrected via a simple proportional feed-back gain $k_{c}$

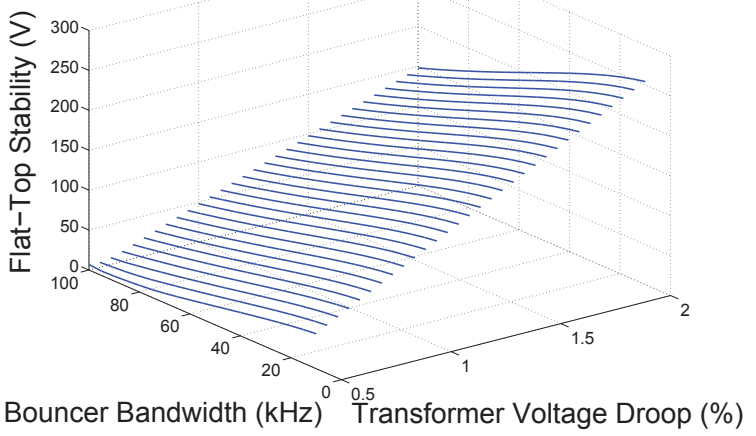

Fig. 7. Influence of voltage droop in the transformer and active bouncer bandwidth on the FTS

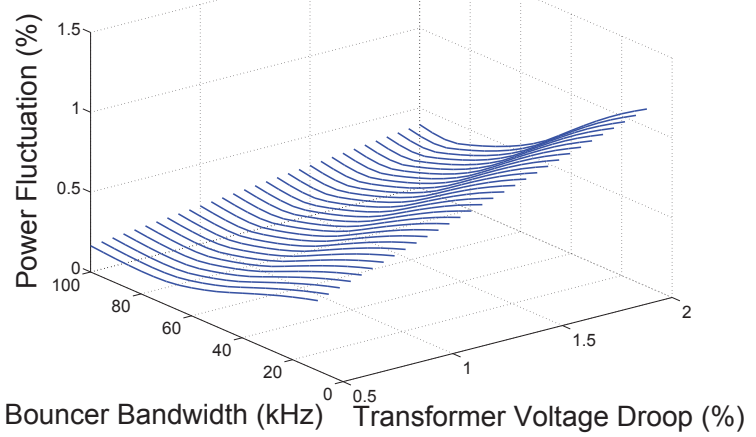

Fig. 8. Influence of voltage droop in the transformer and active bouncer bandwidth on the $\mathrm{AC}$ power fluctuation

to attenuate the pulse transformer voltage droop effect. The DPAB DC-Bus voltage level has been fixed to $V_{A B}=1 \mathrm{kV}$ and the nominal charger voltage to $V_{c h}=15 \mathrm{kV}$.

The optimization objective consists in minimizing the main capacitor bank $C_{\text {main }}$, the active bouncer bandwidth, power fluctuation and FTS. Furthermore the pulse efficiency (difference between the ideal square-shaped pulse and the real one including dynamics) and the PT voltage droop $V_{t_{-} d r o o p}$ (to relax the PT design) are maximized. Equation (1) shows the objective function and in (2) the pulse efficiency definition is presented. The optimization variables are : the gain $k_{c}$, the

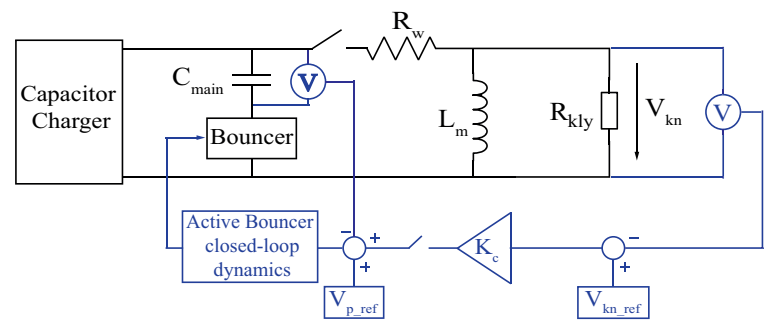

Fig. 9. Modulator model considered for the integrated optimization design. 
active bouncer damping factor and closed-loop bandwidth, the percentage of voltage droop in the transformer $\left(R_{w}\right.$ and $\left.L_{m}\right)$ during the pulse and the main capacitor bank $C_{\text {main }}$ size. The constraints are a maximum power fluctuation value of $5 \%$ and a minimum $F T S=0.85 \%$. The results from this illustrative optimization are shown in table II.

$$
\begin{gathered}
f(x)=\min \left(\frac{C_{\text {main }} * \omega_{\text {bouncer }} * \Delta P * F T S}{\eta_{\text {pulse }} * V_{t \_d r o o p}}\right) \\
\eta_{\text {pulse }}=\frac{E_{\text {ideal_pulse }}}{E_{\text {real_pulse }}}
\end{gathered}
$$

\section{DPAB DESIGN ASPECTS}

\section{A. DPAB output filter}

The primary objective of the DPAB output filter is to attenuate the switching harmonics which may appear on the secondary voltage $V_{s}$. A second order $L C$ filter can be computed considering that the PT transfer function can greatly help in the attenuation action. Furthermore the DPAB is treating a fraction of the total voltage applied to the primary of the PT, meaning that its switching harmonics are even further attenuated. However, at the moment of closing the main switch $M_{s w}$ to create the voltage pulse, a large load current equal to $I_{s} * \frac{1}{R t}$ suddenly starts flowing into the DPAB output filter capacitor, which tends to discharge to negative voltages. The active bouncer is not able to compensate this current perturbation while the filtering inductance current is below the load current. The peculiarity of designing a high performance active bouncer consists in not only obtaining a high voltage bandwidth, but also maximizing the immunity to the external perturbing current, as the load is not passive. In other terms, the current bandwidth has also to be considered.

To face this issue a multi-phase converter offers great advantages, since the current bandwidth is greatly increased due to the parallelization of several output filter inductances. During the rising up of the load voltage and current, the DPAB can be operated such that all inductances are connected in parallel to force the load current flowing through the DC-Bus $C_{A B}$, instead of flowing through the output filter capacitor.

Finally, one has to notice that during the two distinct operating modes, charging and pulsing, the modulator circuit changes, and the transfer function seen by the DPAB is changing accordingly. This complicates the design of the DPAB output filter since during the charging process the transfer function between primary voltage and DPAB output voltage is 1 , and the PT does not contribute to the harmonics attenuation

TABLE II

SIMPLIFIED INTEGRATED OPTIMIZATION RESULTS.

\begin{tabular}{ccc}
\hline \hline$\omega_{\text {bouncer }}$ & 40 & $k \mathrm{~Hz}$ \\
$\delta_{\text {bouncer }}$ & 0.85 & \\
$V_{t_{\text {droop }}}$ & 2.7 & $\%$ \\
$C_{\text {main }}$ & 200 & $\mu \mathrm{F}$ \\
\hline
\end{tabular}

any more. Therefore, in the charging phase the DPAB output filter must be more damped compared to the requirements in pulsing mode. With the purpose of designing a suitable filter for both operating modes, a resistively damped LC-4C filter can be retained.

\section{B. DPAB power design}

1) Capacitor bank size: The dimensioning of the DPAB capacitor bank $C_{A B}$ shall consider the cycles depicted in Fig. 2. The capacitor bank must be designed such that enough voltage $V_{A B}$ is guaranteed during the whole cycle for a correct operation of the DPAB. Considering a maximal voltage droop of $20 \%$ on $V_{A B}$, the capacitor value $C_{A B}$ can be analytically computed as shown in (3).

$$
C_{\text {bouncer }}=\frac{I_{c h} * V_{b_{\max }} * \frac{\left(1 / R E P R-t_{\text {flat }}\right)}{2}}{V_{b_{\max }}^{2}-\left(0.8 * V_{b_{\max }}\right)^{2}} ;
$$

Notice that no losses in the DPAB have been considered here. In the real case, in order to fully recharge the active bouncer capacitor bank after every cycle, the losses in the system (caused by the damping resistor of the filter, switching losses, etc.) must be compensated either by the insertion of a small DC supply to recharge $C_{A B}$ or by a special control drawing the necessary power losses from the main charger.

2) DPAB Converter topology: In order to achieve a 4quadrant operation respecting the voltage and current bandwidths requirements, a multiphase interleaved H-bridge topology is proposed and illustrated in Fig. 10. The required number of phases depends on the large signal current bandwidth (previously discussed) and the maximum switching frequency allowed on each IGBT-based H-bridge module (to achieve the required closed-loop voltage bandwidth and harmonics attenuation).

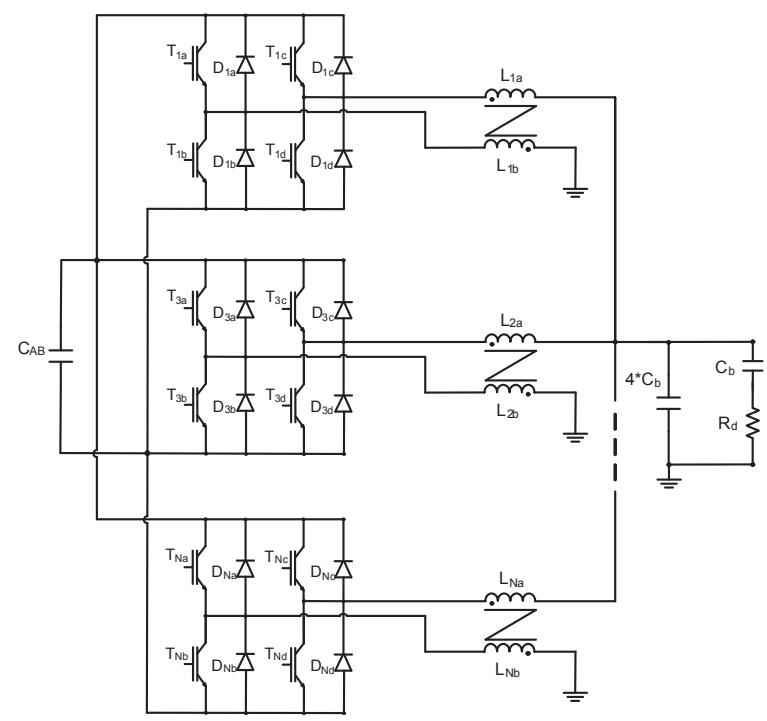

Fig. 10. Modulator model considered for optimization purposes. 
TABLE III

CLIC Modulator's OUtPut PULSE SPECIFICATION

\begin{tabular}{|c|c|}
\hline Pulse Transformer & Active Bouncer \\
\hline Equiv. Capacitance $: 86.7 n F$ & DC bus : $1000 \mathrm{~V}$ \\
Leakage inductance $: 3 \mu \mathrm{H}$ & Filter Inductance $: 25 \mu \mathrm{H}$ \\
Winding resistance $: 14.8 \mu \Omega$ & Filter Capacitor $: 20 \mu \mathrm{F}$ \\
Magnetizing inductance $: 60 \mathrm{mH}$ & Damping resistance $: 560 \mu \Omega$ \\
Transformation ratio $: 10$ & Capacitor bank $C_{A B}: 2 \mathrm{mF}$ \\
Klystron resistance $: 9.375 \Omega$ & Number of phases $: 5$ \\
\hline
\end{tabular}

\section{DPAB Control Strategy}

Considering the dramatic difference between the two voltage transfer functions in each operating mode (pulsing : transfer function between $V_{k n}$ and active bouncer output voltage; charging : transfer function between $V_{p}$ and active bouncer output voltage), a control strategy using different voltage controllers specifically optimized for each operating mode is proposed. The selected controllers consist in two generalized polynomial RST digital controllers [3] which are able to compensate the dynamics and handle the important delays of the system. Due to the multi-phase interleaving solution proposed for the DPAB topology, an additional low gain current sharing loop, able to balance the current among the different phases, has been included as well. Figure 11 illustrates the proposed control structure.

\section{DPAB PERFORMANCES EVALUATION ON CLIC SPECIFICATIONS BY SIMULATION}

With the purpose of validating the correct operation and evaluate the performances of the DPAB, a simulation considering CLIC specifications has been carried out. A primary voltage of $V_{p}=V_{c h}=15 \mathrm{kV}$ and a modulator main capacitor bank of $C_{A B}=150 \mu \mathrm{F}$ (in order to obtain a discharge of $1600 \mathrm{~V}$ during the pulse) have been considered. Preliminary transformer parameters (referred to the primary) and the considered active bouncer, are shown in table III. The results are shown in Fig.12, and show a maximum AC power fluctuation of $2.2 \%$ and a FTS of $0.9 \%$.

\section{CONCLUSION}

A new dual purpose active bouncer system for klystron modulators allows output pulsed voltage regulation, constant

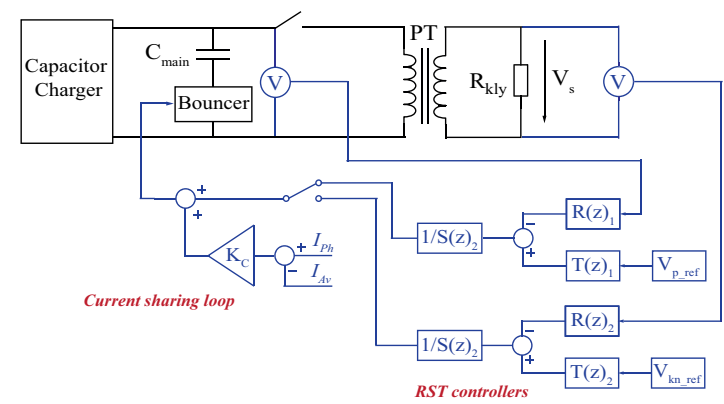

Fig. 11. DPAB control structure; two controllers for two operating modes.
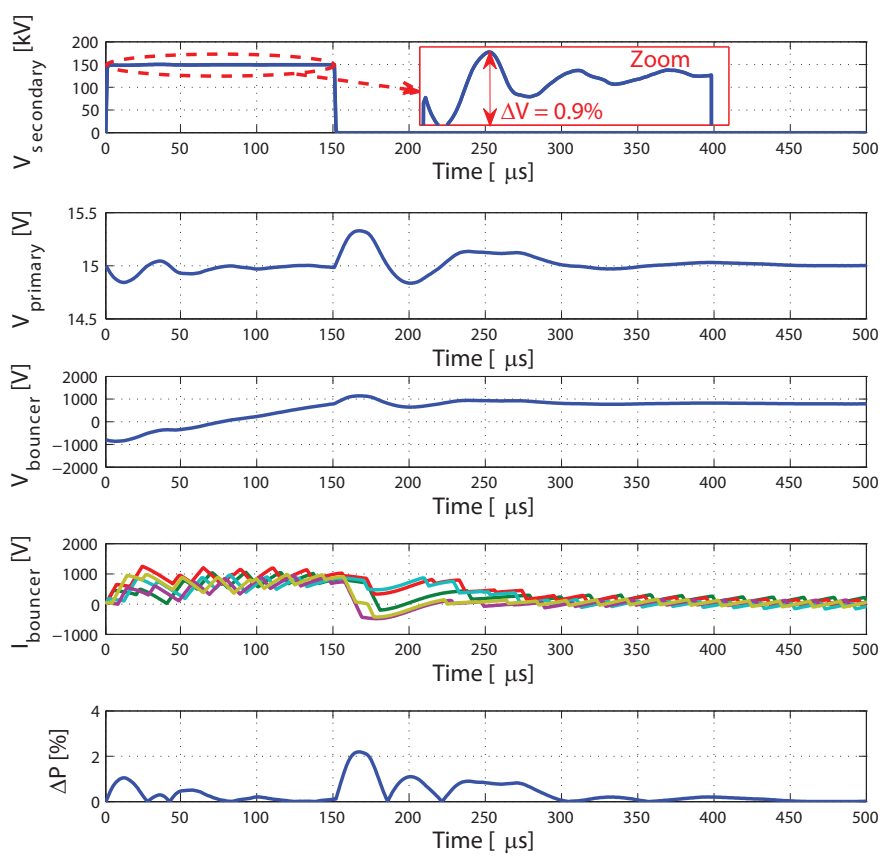

Fig. 12. Results of the DPAB-based modulator for CLIC specifications.

power operation of the modulator and the use of a standard constant voltage \ constant current power supply as a charger. The design compromises between all modulator subcomponents have been presented and the need for a global optimization approach seems to be very important. Preliminary numerical simulations of a modulator based on CLIC specifications, equipped with the proposed DPAB system, show promising results. This work demonstrates that the proposed topology is a promising candidate for the final CLIC modulator design. Ongoing works are focusing on comparing the DPAB topology with the one proposed in Fig. 1b).

\section{REFERENCES}

[1] CERN - European Organization for Nuclear Research. The CLIC Programme towards a staged $e^{+} e^{-}$Linear Collider exploring the Terascale, CLIC Conceptual Design Report, 2012.

[2] R. Corsini. Status and Future of the Clic Study, Proceedings of LINAC2012, Tel-Aviv, Israel.

[3] D. Aguglia, C. A. Martins, M. C. Bastos, D. Nisbet, D. Siemaszko, E. Sklavounou and P. Viarouge. Klystron Modulator Technology Challenges for the Compact Linear Collider (CLIC), 2011, pp. 1413-1421.

[4] D. Aguglia and E. Sklavounou. Klystron Modulators Capacitor Chargers Design Compromises for AC Power Quality Increase of the Compact Linear Collider (CLIC), SPEEDAM 2012, pp. 1535-1541.

[5] G.N. Glasoe and J.V. Lebacqz. Pulse Generators, New York : McGrawHill, 1948. MIT Radiation Laboratory Series.

[6] Coleman, T.F. and Y. Li. An Interior, Trust Region Approach for Nonlinear Minimization Subject to Bounds, SIAM Journal on Optimization, Vol. 6, pp. 418 - 445, 1996.

[7] Coleman, T.F. and Y. Li. On the Convergence of Reflective Newton Methods for Large-Scale Nonlinear Minimization Subject to Bounds, Mathematical Programming, Vol. 67, Number 2, pp. 189 - 224, 1994.

[8] Powell, M.J.D. A Fast Algorithm for Nonlinearly Constrained Optimization Calculations, Numerical Analysis, ed. G.A. Watson, Lecture Notes in Mathematics, Springer Verlag, Vol. 630, 1978.

[9] Y. D. Landau, G. Zito. Digital control systems : design, identification and implementation, Springer-Verlag London limited, 2006, 484p. 